Those practitioners who have encountered the progress of this epidemic need not fear that they will find in the aspect and mortality of any other disease, although it may be of more famous name, features more formidable and success more disheartening. Yours, respectfully,

Manchester; December 15th, 1831.

John ROBERTon.

\title{
HEMICRANIA.
}

On one of those Affections designated Migrane (Megrim), or Hemicrania. By M. Piorry, M.D.

THE foregoing subject occupies some twenty pages of M. Piorry's late work. The author observes that, under the term hemicrania, authors have arranged several different disorders. Chaussier and his followers have pronounced it a neuralgia of intermittent or continued character, and of greater or less intensity. The author agrees with this opinion ; but observes, that hemicrania cannot always be considered as a facial neuralgia. It differs, he remarks, materially from tic douloureux, and also from the pains occasioned by carious teeth. If, indeed, says he, we understand by the word hemicrania, a pain seated on one side of the head, almost all neuralgia of this part must come under the designation, since few of them attack more than one side of the body, or pass the median line. But if, by hemicrania, we mean a specific affection, having its seat in or near one of the eyes-differing from all the other neuralgia - followed by sickness and generally relieved by vomiting - ceasing after. a single paroxysm, not to return for a considerable time in general-then. we must separate hemicrania from the other neuralgix with which it has. hitherto been confounded, in order to study its symptoms, its signs, and its treatment. The author affirms that he has paid great attention to this disease-and thinks that investigation of it throws some light on the neuroses in general.

The complaint in question is conceived by the author to be a neurosis, or rather a neuralgia of the iris, which, at first bounded to that membrane, or, more properly speaking, to its nerves, extends to a number of other nervous branches, and is characterised by disturbance of vision, succeeded by pain in the eye, or on the surface of the cranium, by sickness, and by vomiting. This ophthalmic neurosis is observable among. people whose sight is weak, to whom strong light is disagreeable, and dark rooms pleasant - who study and write much - who lead a sedentary life-and among workmen who are much occupied with the inspection of minute bodies.

On the other hand, we rarely see this complaint in people who lead an active life in the open air-who are habitually exposed to a strong: light $\longrightarrow$ and who do not exercise the eyes much. It occurs chiefly, according to the experience of the author, under two opposite conditions. of the stomach-a state of too much repletion, and too great abstinence. In people, in these conditions, a very slight exertion of the eyes will often bring on the ophthalmic neuralgia. A physician of the author's acquaintance generally experienced an attack of this complaint every time: 
that he read a lecture on medicine. Whenever he left off lecturing, or at least the reading of his lectures, he ceased to have the hemicrania ; and whenever he resumed the avocation, the disorder returned. It was remarked that these lectures were delivered on a full stomach directly after dinner.

The hour of attack, however, is not confined to any particular period - sometimes immediately after the application of the exciting causesometimes not till after several hours, but generally within the twenty-four hours. At the moment of the invasion, the sight becomes less clear, and there appears a kind of black speck in the centre of the eye, which gradually enlarges and spreads to the other parts of the organ, still partially surrounded by the arc of a luminous circle, of different colors in different individuals. After a time, this dark centre and sparkling circle begin to grow less distinct, and at last break up and disappear, with return of vision. These phenomena rarely take place, except in one eye. Thus far there is no pain experienced ; but only a kind of stupor, with some derangement of vision and heaviness of head. But after a longer or shorter interval, some darting pains are felt in the eye and temple, and the least pressure on the ball of the eye causes much suffering. The patient complains that the globe of the eye feels too full, attended with pulsation of a dolorous kind. These sensations are not uniformly pungent, but remit and exasperate, like colic or spasmodic pains in other parts. The duration of these attacks varies from some hours to two or three days.

Meantime the senses of hearing, tasting, and smelling, are more or less deranged with that of sight. The eyelids become red and tumefied-the access of light is insufferable-the least noise offends the ear-and the taste for food is quite perverted. The sensorial functions are undisturbed; except that there is a greater tendency to sleep than usual.

Such are the phenomena of cases the most simple; but very frequently the stomach participates in the complaints of the eye. Soon after the ophthalmic symptoms commence, eructations from the stomach takc place, followed by some nausea, and even by vomiting of the food lately taken, or, if empty, of glairy mucus. In severe cases the stomach is not the only organ which sympathizes with the eye. Often one side of the tongue or of the fice, or one of the upper or lower extremities, experience a kind of painful tremor or vibration, like that which is felt after striking the cubital nerve, at the elbow, against some hard body. In general, the heart, lungs, and intestinal canal, remain free from any morbid affection. A restorative sleep usually terminates the paroxysm, after several hours', or two or three days' duration. A heaviness of the head is felt for a day or two after the cessation of the hemicrania. The recurrence of the malady is uncertain ; and generally only when the exciting causes are strongly applied. Our author knows a female who has six months' interval between the attacks, provided she does not read within two hours after taking food. If she uses not this precaution, she is sure of an attack immediately after her transgression. In certain individuals the attack is periodical, returning every eight days, every month, or every two or three months, with considerable regularity. In others, there is no fixed period for relapse. 
The prognosis in this curious complaint, is generally favorable, as far as life is concerned ; but if the complaint proves rebellious, it renders life miserable. When it fails to be cured, the paroxysms return at shorter and shorter intervals, till life becomes one scene of suffering. Our author has not been able to find any information respecting the pathological anatomy of hemicrania ; but suspects that the scalpel will not reveal any lesion of structure in the brain or membranes to account for the phenomena. This neuralgia, he imagines, is too fugitive, subject to too many remissions or intermissions, to leave organic traces that might be detected by the eye. He justly observes that the dissecting room is not the only place where we may study pathology. The sick-room will often afford us much useful information in this respect. An examination of the eye, during the attack of hemicrania, shows the pupil strongly contracted, and consequently the iris put upon the stretch, with redness of both palpebræ. From these phenomena $M$. Piorry concludes that, in hemicrania, an exciting cause acts on the retina and iris-the nervous action is modified-a kind of struggle takes place, evinced by oscillations and vibrations, with the luminous circle, dark spots, \&c. before alluded to. In time, the fifth pair of nerves participate in the morbid action, and ultimately other organs and parts with which the fifth pair communicate.

TrEaTMENT.-Our author considers himself as very successful in the treatment of this painful malady. His first indications are to arrest the development of the series of symptoms constituting hemicrania-and to mitigate its accessions (calmer ses accés). It is at the moment of its commencement that the course of the malady is most easily checked. At this period all causes of excitation in the optic nerves and tissues should be removed. The patient is to be completely excluded from light and noise. This abduction of all stimulus will sometimes induce early sleep, and check the paroxysm. It is at this early period that the application of belladonna has occasionally succeeded in preventing the pain. The author and his colleague, M. Trousseau, cause the remedy to be rubbed on the temples with the greatest success. M. Piorry dilutes the belladonna with a little water so as to form a kind of syrup, which is rubbed on the palpetra as well as on the ternples. The author uses only a very small quantity of the remedy, from one to three or four grains. He avers that he hardly ever fails to check the paroxysm by this means. It is to be borne in mind that, on the succeeding day after the application of the belladonna, the pupils will be greatly dilated, and vision disturbed. But this effect and inconvenience are only temporary. The author has not employed belladonna internally. He has used opium, but with very indifferent effects. M. Piorry observes that the march of hemicrania may often be arrested by raising excitement in the stomach, by means of stimulants, as wine, spices, food, \&c. A very smart stimulation to the feet will sometimes have the same effect. In case of failure, each symptom can only be combated by the most probable means. Quietude and darkness-cold applied to the eye affected-vomiting by means of large ingurgitation of warm water, will mitigate the pain, and somewhat curtail the paroxysms.

The prevention of a return then becomes the great indication. The causes already onumerated are to be avoided, and especially all exercise 
of the eye in reading during the operation of digestion. After this process is finished in the stomach, the patient need not fear to engage in study or other exercise. Exposure to a strong light, however, and sudden transition from a dark to an illuminated room, are dangerous. Sometimes a local plethora predisposes to hemicrania; then abstraction of blood is proper. On the other hand, where the patient leads a sedentary life, and is debilitated, we ought to prescribe nourishing food, which is the best of all tonics. Great attention to the bowels is necessary, since constipation often renews the attack. It is remarkable that the author appears to have had but little experience of the efficacy of quinine in this complaint -and of arseric he makes no mention at all. Yet these are the most potent of all remedies in the disease under consideration. We have met with the complaint very often-indeed, it is by no means unfrequent in this metropolis, among urtists and others who lead a sedentary life, using the eye much, and the muscles of the body little. In these people, we have first cleared the bowels, and then given a sudorific at bed-time, with a good dose of colchicum and Batley's liquor opii sedativus. After this the quinine, arsenic, or both united, have seldom failed to put a sudden stop to the complaint. Quinine in small doses, for some weeks afterwards, is necessary to prevent relapses.-Med.-Chir. Rev.

BOSTON MEDICAL AND SURGICAL JOURNAL.

B O S T O N, MAR C 21, 1832 .

\section{ALLEGED MAL-PRACTICE.}

THE following notice appeared in the Litchfield (Connecticut) Enquirer of Thursday last.

"The Superior Court of this County, Judge Daggett presiding, closed its sessions on Tuesday night last, after an arduous session of fifteen days. Besides the criminal trials, there was a number of civil cases disposed of, among which was the following :-

"Harriet A. London ve. Dr. Asahel Humphrey.-This was an action for mal-practice in performing the operation of vaccination upon the left arm of the plaintiff, in March, 1829. It appeared that the defendant and sundry other physicinns had made a contruct with the Board of Health of the town of Salisbury, to vaccinate the inhabitants of that town-that the physicians divided the town into districts to enable them to perform with facility the duty assigned them-that the plaintiff resided in the district confined to the care of the defendant; that the defendant employed one Rollin Sprague, a young student of medicine, to attend to the business for him as his agent, and that Sprague vaccinated the plaintiff, and made two punctures just above the elbow joint in a very unusual place-the effect of which was immediate, and was attended with the most serious consequences, indicating, in the opinion of Professor Hubbard and others, with unerring certainty, that a nerve had been wounded. It was proved : that the injury was probably incurable. The case underwent an elahorate investigation, and the jury rendered a verdict for the plaintiff- $\$ 400$ damages." 